\title{
An Egyptian study to assess the accuracy and reliability of CAD-RADS CT coronary angiography algorithm in the evaluation of coronary artery disease
}

\author{
Mena E. Y. Ekladious* ${ }^{*}$, Mounir Sobhy Guirguis, Ahmed Mohammed Haggag and Ahmed S. Abdelrahman
}

\begin{abstract}
Background: Multidetector computed tomography angiography (MDCT) is a non-invasive examination for coronary artery disease. Coronary artery disease reporting and data system (CAD-RADS) is a structured reporting system that successfully facilitated communication with clinicians. Our study aimed to assess the accuracy as well as the agreement of the CAD-RADS system with the conventional angiography results.

Results: 48 patients were enrolled in this prospective study, all patients underwent MDCT coronary angiography and conventional coronary artery angiography. An excellent inter method agreement between coronary CT angiography (CCTA) and conventional coronary angiography was noted for the left main trunk (LMT) with $k=1(p<0.001)$. An excellent inter method agreement was found for the proximal, mid- and distal segments of the left anterior descending artery (LAD) and the second diagonal segment, with $k=1,0.8420 .886$ and 0.886 , respectively $(p<0.001)$. A good agreement was noted at the first diagonal segment with $k=0.765(p<0.001)$. An excellent inter-method agreement was found for the proximal, mid- and distal segments of the left circumflex artery (LCX) and the obtuse marginal branch, with $k=0.838,0.846,1$ and 0.846 , respectively $(p<0.001)$. An excellent agreement was found for the proximal and mid-segments of the right coronary artery (RCA) and the posterior descending artery, with $k=1$ for all segments $(p<0.001)$, yet a good agreement was noted at its distal segment with $k=0.782(p<0.001)$. The overall per-patient sensitivity, specificity and accuracy of the CT coronary angiography were $92.9 \%, 90 \%$ and $91.7 \%$ respectively.
\end{abstract}

Conclusions: The CAD-RADS algorithm and invasive coronary angiography agreed perfectly; thus, CT coronary angiography can be used as the first screening test and the invasive coronary angiography can be spared for cases requiring intervention.

Keywords: Coronary CT angiography (CCTA), Invasive coronary angiography (ICA), CAD-RADS, Coronary artery disease

\section{Background}

Coronary artery disease (CAD) is considered to be the most common cause of death worldwide rendering the proper diagnosis and appropriate management of utmost

*Correspondence: menaerian@yahoo.com

Radiology Department, Faculty of Medicine, Ain Shams University, Cairo, Egypt importance in healthcare management across the globe [1]. Conventional angiography is considered to be the gold standard for the diagnosis, yet it is an invasive procedure that may imply associated complications, it also requires a short hospital stay which adds to the financial and psychological burden of the patient [2]. That led to the widespread use of coronary computed tomography angiography (CCTA) as a non-invasive investigation for coronary artery disease, it proved to be of high negative 
predictive value and thus has become the initial investigation of coronary artery disease especially in low-risk patients [3].

To unify the reporting system and to link diagnostic data with patient management, Curye et al. [4] developed coronary artery disease reporting and data system (CADRADS). Many previous studies have assessed the accuracy of coronary $C T$ angiography yet, very limited studies considered the CAD-RADS classification system per se [5-7] and although the CAD-RADS system is widely used by many institutes, it is still not widely accepted officially as a reporting system for coronary $\mathrm{CT}$ angiography across the globe [8]. Structured reporting has emerged as the best choice for reporting, attempting to provide a precise answer to a clinician's question and sharing in the patient management by providing the appropriate next step management based on radiological imaging [9].

This study aimed to assess the accuracy as well as the agreement of the CAD-RADS system with the conventional angiography results which will support the concept of replacing the invasive conventional angiography by $\mathrm{CT}$ angiography especially at stable cases.

\section{Methods}

This prospective study was done at our institution from October 2020 to August 2021, it included 48 patients, 28 males (58\%) and 20 females (42\%) with a mean age of $49 \pm 9.5$ years. The study was conducted according to the stipulations of the ASU ethical and scientific committee, informed written consents were obtained from all the patients. Inclusion criteria were patients 30 to 80 years who were presented with stable chest pain. Exclusion criteria were patients with arrhythmia, contrast medium hypersensitivity or allergy, patients with chronic kidney disease, pregnant and lactating females as well as patients with high calcium scores (Agatston score $>1000$ ).

All patients underwent MDCT coronary angiography followed by conventional coronary artery angiography study with 1-2 day duration.

\section{Technique of CT coronary angiography}

MDCT coronary angiography was performed with an ECG-gated 128-multidetector CT scanner (GE optima). Beta-blockers was administered for the patient with a heart rate $>65$ BPM. Agatston calcium score was done then a timing bolus (using 10 to $20 \mathrm{ml}$ contrast) is injected to detect the proper timing for optimal contrast opacification in the axial image at a level just superior to the ostium of the left main artery. Nitroglycerine $0.4 \mathrm{mg}$ sublingually was administered immediately before contrast injection to dilate the coronary vessels. During CCTA acquisition, $80-\mathrm{ml}$ iodinated contrast was injected using a triple-phase contrast protocol: $60-\mathrm{ml}$ contrast medium, followed by $40 \mathrm{ml}$ of a 50:50 mixture of contrast medium and saline, followed by a $50-\mathrm{ml}$ saline flush. Retrospective electrocardiogram-gated helical contrastenhanced CCTA was performed, with scan initiation $20 \mathrm{~mm}$ above the level of the left main artery to $20 \mathrm{~mm}$ below the inferior myocardial apex. The scan parameters were: $100-140 \mathrm{kV}, 800 \mathrm{~mA}, 128 \times 0.6-\mathrm{mm}$ collimation and $0.6-\mathrm{mm}$ pitch. The total scanning time was approximately 9-11 s. After scan completion, multiphasic reconstruction of the CCTA scans was performed using a dedicated workstation (optima GE medical system S.C.S., France), with reconstructed images, curved multi-planar reformation and volume-rendered reconstruction.

Imaging interpretation was done by two conjoint radiologists with 4 and 5 years of experience in cardiac imaging (there was no controversy in their mutual reporting of findings). They primarily assessed dominance, plaque characterization, patency and degree of stenosis guided by the CAD-RADS template where the coronary stenosis was graded into the following categories: CAD-RAD $0=0 \%$ degree of stenosis, CAD-RAD $1=0 \%$ to $24 \%$ degree of stenosis, CAD-RAD $2=25 \%$ to $49 \%$ degree of stenosis, CAD-RAD $3=50 \%$ to $69 \%$ degree of stenosis, CAD-RAD $4=>70 \%$ degree of stenosis, CAD-RAD $5=$ Total occlusion. Modifiers are implemented for further illustration; modifier N: nondiagnostic, modifier S: stent, modifier G: graft, modifier V: vulnerability (used when at least two features of high-risk plaque are present, high-risk features are: low attenuation plaque (less than 30 Hounsfield Units), positive remodeling, spotty calcification, low attenuation plaques with peripheral high attenuation "napkin ring sign") (Table 1) [4].

\section{Invasive conventional angiography (ICA) procedure}

Selective ICA was performed by standard trans-femoral arterial catheterization. A minimum of eight projections was obtained (a minimum of 5 views for the left coronary artery system and a minimum of 3 views for the right coronary artery system). Due to differences in cardiac position, the angles of projection for ICA showed slight differences among patients. All ICA images were interpreted independently by a reader who was blinded to CCTA results. The ICAs were quantitatively evaluated for coronary artery stenosis.

\section{Statistical analysis}

Statistical analysis was performed using the statistical software: SPSS statistical package version 21 (SPSS Inc., Chicago, IL). Numerical data were expressed as mean and standard deviation or median and range as appropriate. Qualitative data were expressed as frequency and percentage. CT coronary angiography and conventional features that were analyzed included the significance of 
Table 1 CAD-RADS classification

\begin{tabular}{lll}
\hline CAD-RADS category & Degree if maximum coronary stenosis & Management \\
\hline 0 & $0 \%$ & None \\
1 & $0 \%$ to $24 \%$ & None \\
2 & $25 \%$ to $49 \%$ & None \\
3 & $50 \%$ to $69 \%$ & Functional assessment \\
$4 \mathrm{a}$ & One or two vessels $>70 \%$ & Conventional angiography \\
& & or functional assessment \\
$4 \mathrm{~b}$ & LMT > $50 \%$ or three vessels $>70 \%$ & Conventional angiography \\
5 & Total occlusion & Conventional angiography \\
& & and/or viability assess- \\
& ment
\end{tabular}

Modifiers are implemented for further illustration; modifier N: nondiagnostic, modifier S: stent, modifier G: graft, modifier V: vulnerability

the lesion. $P$-value was calculated. The confidence interval was set to $95 \%$ and the margin of error accepted was set to $5 \%$. So, $p$ value $<0.05$ was considered significant. Kappa coefficient value $(k)$ was measured and interpreted as follows: $k<0$ : no agreement, $k=0.01-0.20$ : slight agreement, $k=0.21-0.40$ : fair agreement, $k=0.41-0.60$ : moderate agreement, $k=0.61-0.8$ : good agreement, $k=0.81-1.00$ : excellent agreement. Diagnostic performance was measured in terms of specificity, sensitivity, accuracy, positive and negative predictive values.

\section{Results}

The study involved 48 patients, 28 males (58\%) and 20 females (42\%) with an age range from 30 to 69 years $($ Mean $=49 \pm 9.5)$. Forty patients $(83.3 \%)$ showed right dominance, six patients (12.5\%) showed left dominance and two patients (4.2\%) showed co-dominance.

\section{Per-artery analysis}

As regards the agreement of the findings detected by CT angiography and conventional angiography at the left main trunk (LMT), there was an excellent agreement between CT coronary angiography and conventional coronary angiography as regarding the 46 non-significant (95.8\%) and $2(4.2 \%)$ significant lesions at each of the proximal, mid- and distal segments. An excellent agreement was noted at the proximal segment with kappa coefficient $k=1$ and $(p<0.001)$ (Table 2).

At the left anterior descending artery (LAD), there was complete agreement between the CT coronary angiography and the conventional coronary angiography regarding 38 non-significant $(79.2 \%)$ and $10(20.8 \%)$ significant lesions at the proximal segment of the LAD (Fig. 1), as well as regarding 48 (100\%) non-significant and 0 significant lesions at D1 and D2 branches. Regarding the midsegment of the LAD, there was a difference between the CT coronary angiography and the conventional coronary angiography findings, as two lesions were reported as severe (CAD-RADS-4) by the CTA which were found to be normal by the conventional angiography (Fig. 2). Regarding the distal segments of the LAD, there was also a difference between the CT coronary angiography and the conventional coronary angiography findings as two significant lesions were diagnosed by the conventional angiography which was not detected by CTA. Excellent agreements were noted at the proximal segment, midsegment, distal segment and D2 segment with $k=1$, $0.842,0.886$ and 0.886 , respectively $(p<0.001)$. A good agreement was noted at the D1 segment with $k=0.765$ $(p<0.001)$ (Table 2).

At the left circumflex artery ( $\mathrm{LCx}$ ), there was complete agreement between CT coronary angiography and conventional coronary angiography regarding 44 non-significant $(91.7 \%)$ and $4(8.3 \%)$ significant lesions at its distal segment (Fig. 3). Regarding the proximal segments, there was a difference between CT coronary angiography and conventional coronary angiography findings. Two severe lesions (CAD-RADS 4) were reported by CTA; however, they were confirmed to be mild (CAD-RADS 2) by the conventional angiography. Regarding the mid-segment of LCx and obtuse marginal branch (OM), there were differences between the results of both examinations. Two severe lesions at each were detected by CTA (CADRADS 4) that were proved to be normal by the conventional angiography. Excellent agreements were noted at the proximal segment, mid-segment, distal segment and OM branch with $k=0.838,0.846,1$ and 0.846 , respectively $(p<0.001)$ (Table 2$)$.

At the right coronary artery (RCA), there was complete agreement between the CT coronary angiography and the conventional coronary regarding 46 non-significant (95.8\%) and $2(4.2 \%)$ significant lesions at the proximal segment of the RCA (Fig. 4), the 42 non-significant $(87.6 \%)$ and $6(12.4 \%)$ significant lesions at each of the 
Table 2 Analysis of the coronary vessels findings by the conventional and CT angiography

\begin{tabular}{|c|c|c|c|c|}
\hline & CT angiography & Conventional angiography & Kappa & $p$ value \\
\hline \multicolumn{5}{|l|}{ LMT } \\
\hline Proximal & & & 1 & $<0.001^{*}$ \\
\hline Normal/mild/moderate (CAD-RADS 0-3) & $46(96 \%)$ & $46(96 \%)$ & & \\
\hline Severe/occluded (CAD-RADS 4-5) & $2(4 \%)$ & $2(4 \%)$ & & \\
\hline Mid & & & 1 & $0.001^{*}$ \\
\hline Normal/mild/moderate (CAD-RADS 0-3) & $46(96 \%)$ & $46(96 \%)$ & & \\
\hline Severe/occluded (CAD-RADS 4-5) & $2(4 \%)$ & $2(4 \%)$ & & \\
\hline Distal & & & 1 & $0.001^{*}$ \\
\hline Normal/mild/moderate (CAD-RADS 0-3) & $46(96 \%)$ & $46(96 \%)$ & & \\
\hline Severe/occluded (CAD-RADS 4-5) & $2(4 \%)$ & $2(4 \%)$ & & \\
\hline \multicolumn{5}{|l|}{ LAD } \\
\hline Proximal & & & 1 & $<0.001^{*}$ \\
\hline Normal/mild/moderate (CAD-RADS 0-3) & $38(79 \%)$ & $38(79 \%)$ & & \\
\hline Severe/occluded (CAD-RADS 4-5) & $10(21 \%)$ & $10(21 \%)$ & & \\
\hline Mid & & & 0.842 & $0.001^{*}$ \\
\hline Normal/mild/moderate (CAD-RADS 0-3) & $42(87.5 \%)$ & $44(91.5 \%)$ & & \\
\hline Severe/occluded (CAD-RADS 4-5) & $6(12.5 \%)$ & $4(8.5 \%)$ & & \\
\hline Distal & & & 0.886 & $0.001^{*}$ \\
\hline Normal/mild/moderate (CAD-RADS 0-3) & $48(100 \%)$ & $42(87.5 \%)$ & & \\
\hline Severe/occluded (CAD-RADS 4-5) & $0(0 \%)$ & $6(12.5 \%)$ & & \\
\hline D1 & & & 0.765 & $0.001^{*}$ \\
\hline Normal/mild/moderate (CAD-RADS 0-3) & $48(100 \%)$ & $48(100 \%)$ & & \\
\hline Severe/occluded (CAD-RADS 4-5) & $0(0 \%)$ & $0(0 \%)$ & & \\
\hline D2 & & & 0.886 & $0.001^{*}$ \\
\hline Normal/mild/moderate (CAD-RADS 0-3) & $48(100 \%)$ & $48(100 \%)$ & & \\
\hline Severe/occluded (CAD-RADS 4-5) & $0(0 \%)$ & $0(0 \%)$ & & \\
\hline \multicolumn{5}{|l|}{ LCX } \\
\hline Proximal & & & 0.838 & $<0.001^{*}$ \\
\hline Normal/mild/moderate (CAD-RADS 0-3) & $44(91.5 \%)$ & $46(96 \%)$ & & \\
\hline Severe/occluded (CAD-RADS 4-5) & $4(8.5 \%)$ & $2(4 \%)$ & & \\
\hline Mid & & & 0.846 & $0.001^{*}$ \\
\hline Normal/mild/moderate (CAD-RADS 0-3) & $42(87.5 \%)$ & $44(91.5 \%)$ & & \\
\hline Severe/occluded (CAD-RADS 4-5) & $6(12.5 \%)$ & $4(8.5 \%)$ & & \\
\hline Distal & & & 1 & $0.001^{*}$ \\
\hline Normal/mild/moderate (CAD-RADS 0-3) & $44(91.5 \%)$ & $44(91.5 \%)$ & & \\
\hline Severe/occluded (CAD-RADS 4-5) & $4(8.5 \%)$ & $4(8.5 \%)$ & & \\
\hline $\mathrm{OM}$ & & & 0.846 & $0.001^{*}$ \\
\hline Normal/mild/moderate (CAD-RADS 0-3) & $42(87.55)$ & $44(91.5 \%)$ & & \\
\hline Severe/occluded (CAD-RADS 4-5) & $6(12.5 \%)$ & $4(8.5 \%)$ & & \\
\hline \multicolumn{5}{|l|}{ RCA } \\
\hline Proximal & & & 1 & $<0.001^{*}$ \\
\hline Normal/mild/moderate (CAD-RADS 0-3) & $46(96 \%)$ & $46(96 \%)$ & & \\
\hline Severe/occluded (CAD-RADS 4-5) & $2(4 \%)$ & $2(4 \%)$ & & \\
\hline Mid & & & 1 & $0.001^{*}$ \\
\hline Normal/mild/moderate (CAD-RADS 0-3) & $42(87.5 \%)$ & $42(87.5 \%)$ & & \\
\hline Severe/occluded (CAD-RADS 4-5) & $6(12.5 \%)$ & $6(12.5 \%)$ & & \\
\hline Distal & & & 0.782 & $0.001^{*}$ \\
\hline Normal/mild/moderate (CAD-RADS 0-3) & $40(83 \%)$ & 40 (83\%) & & \\
\hline Severe/occluded (CAD-RADS 4-5) & $8(17 \%)$ & $8(17 \%)$ & & \\
\hline PDA & & & 1 & $0.001^{*}$ \\
\hline Normal/mild/moderate (CAD-RADS 0-3) & $42(87.5 \%)$ & $42(87.5 \%)$ & & \\
\hline Severe/occluded (CAD-RADS 4-5) & $6(12.5 \%)$ & $6(12.5 \%)$ & & \\
\hline
\end{tabular}


Table 2 (continued)

\section{к: kappa coefficient}

*Statistically significant $(p<0.05)$
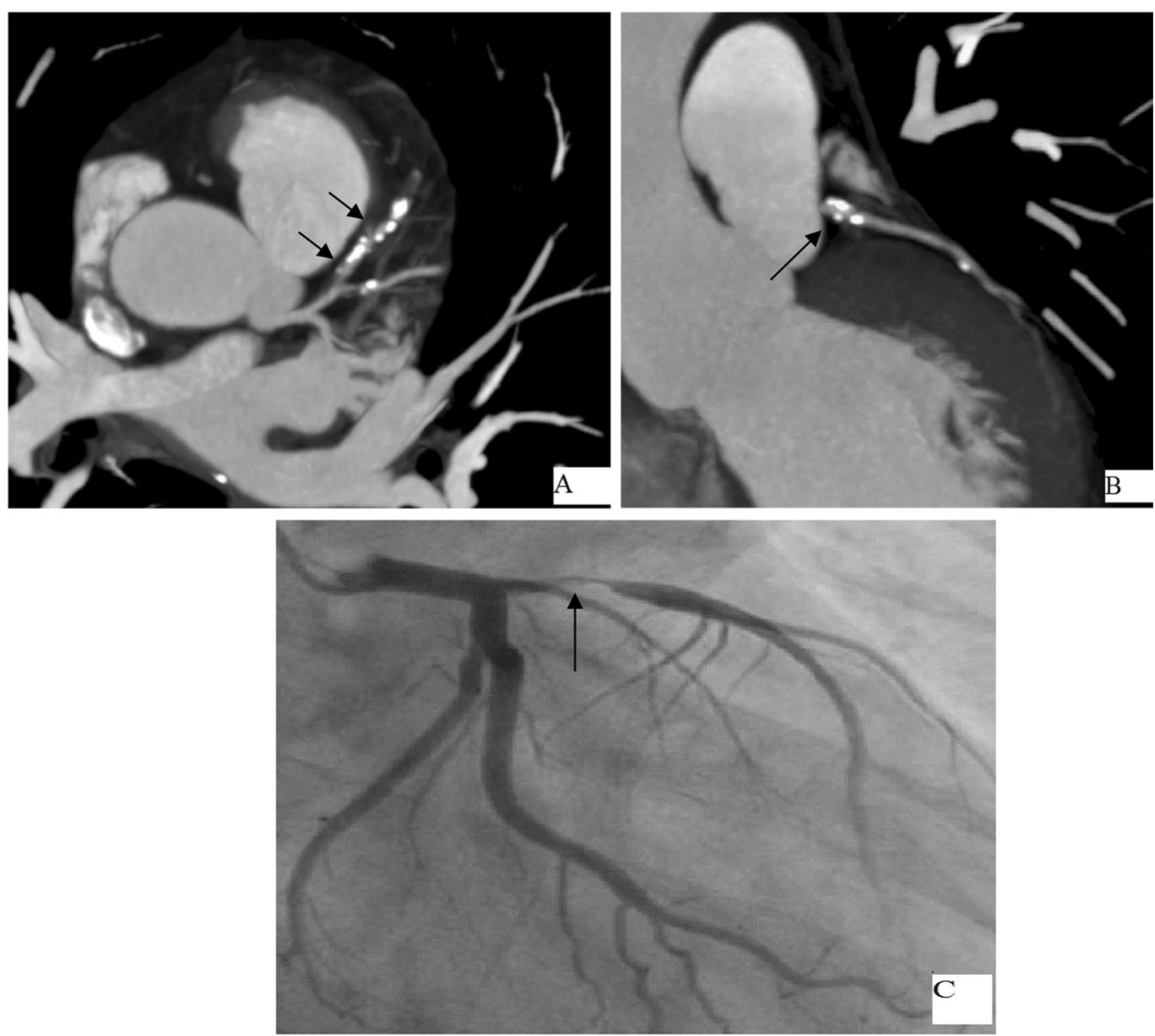

Fig. 1 A 47-year-old male patient presented with chest pain. A, B Axial and Coronal CCTA showing proximal LAD moderate stenotic lesion (CAD-RADS 3) (black arrows). C Conventional angiography shows proximal LAD moderate stenotic segment (arrow) in complete agreement with the CT findings

mid-segment and posterior descending artery (PDA), and the 40 non-significant (83.4\%) and 8 (16.6\%) significant lesions at the distal segment of the RCA. Excellent agreements were noted at the proximal segment, midsegment and PDA branch with $k=1$ for all segments $(p<0.001)$, yet a good agreement was noted at the distal segment with $k=0.782(p<0.001)$ (Table 2$)$.

At the CT examination, $52.1 \%$ of the patients $(25$ patients) showed no plaques, $12.5 \%$ (6 patients) showed non-calcific plaques yet the calcific plaques were detected at $35.4 \%$ of the patients (17 patients); on the other hand, at the conventional angiography, calcified plaques were detected in $27.1 \%$ of the patients (13 patients) yet the remaining $72.9 \%$ of the patients (35 patients) showed no evident calcific plaques. A good agreement was noted at plaques types between the conventional angiography and CT angiography with $k=0.601(p=0.004)$ (Table 3$)$.

\section{Per-patient analysis}

At the overall per-patient analysis, twenty patients showed only non-significant lesions at the conventional angiography, 18 of them (90\%) were the same at the CT angiography but 2 patients (10\%) were overestimated as significant lesions at the $\mathrm{CT}$ angiography.

The other 28 patients with significant lesions at the conventional angiography, 26 of them (92.8\%) also 

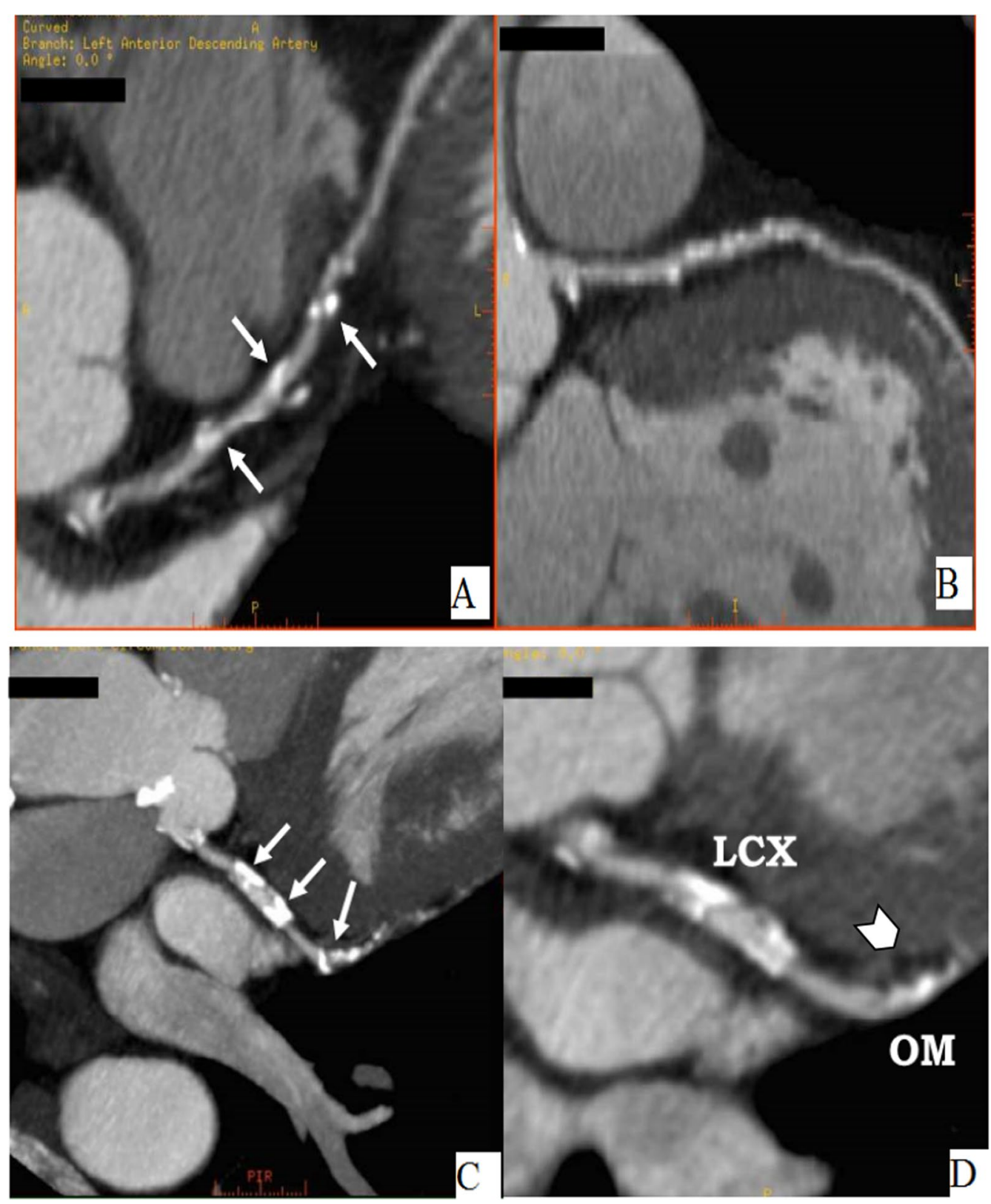

Fig. 2 A 58-year-old male patient presented with attacks of progressive compressing retro-sternal chest pain underwent MSCT. A, B Two curved MPR images of the LAD show multiple calcified plaques, three of them (arrows) show significant stenosis (CAD-RADS 4). C MIP Image and D MPR showing two calcified plaques (arrows) at the LCx and a long calcified plaque at the proximal segment of the OM branch (arrowhead) causing significant stenosis (CAD-RADS 4). E-H Conventional angiography showed that the luminal diameter reduction at proximal and mid-LAD and the proximal LCX segments were over-estimated at CT angiography, and this was attributed to the presence of heavy calcifications that led to blooming effects, (beam hardening artefacts) obscuring the nearby patent lumen (arrows)

showed significant lesions in the CT angiography yet in the remaining patients $(7.2 \%)$ the lesions were missed and underestimated as non-significant at the CT angiography. Thus, the overall per-patient sensitivity, specificity and accuracy of the CT angiography were 92.9\%, 90\% and $91.7 \%$, respectively, with $90 \%$ positive predictive value (PPV) and $92.9 \%$ negative predictive value (NPV) (Table 4, Fig. 5). 


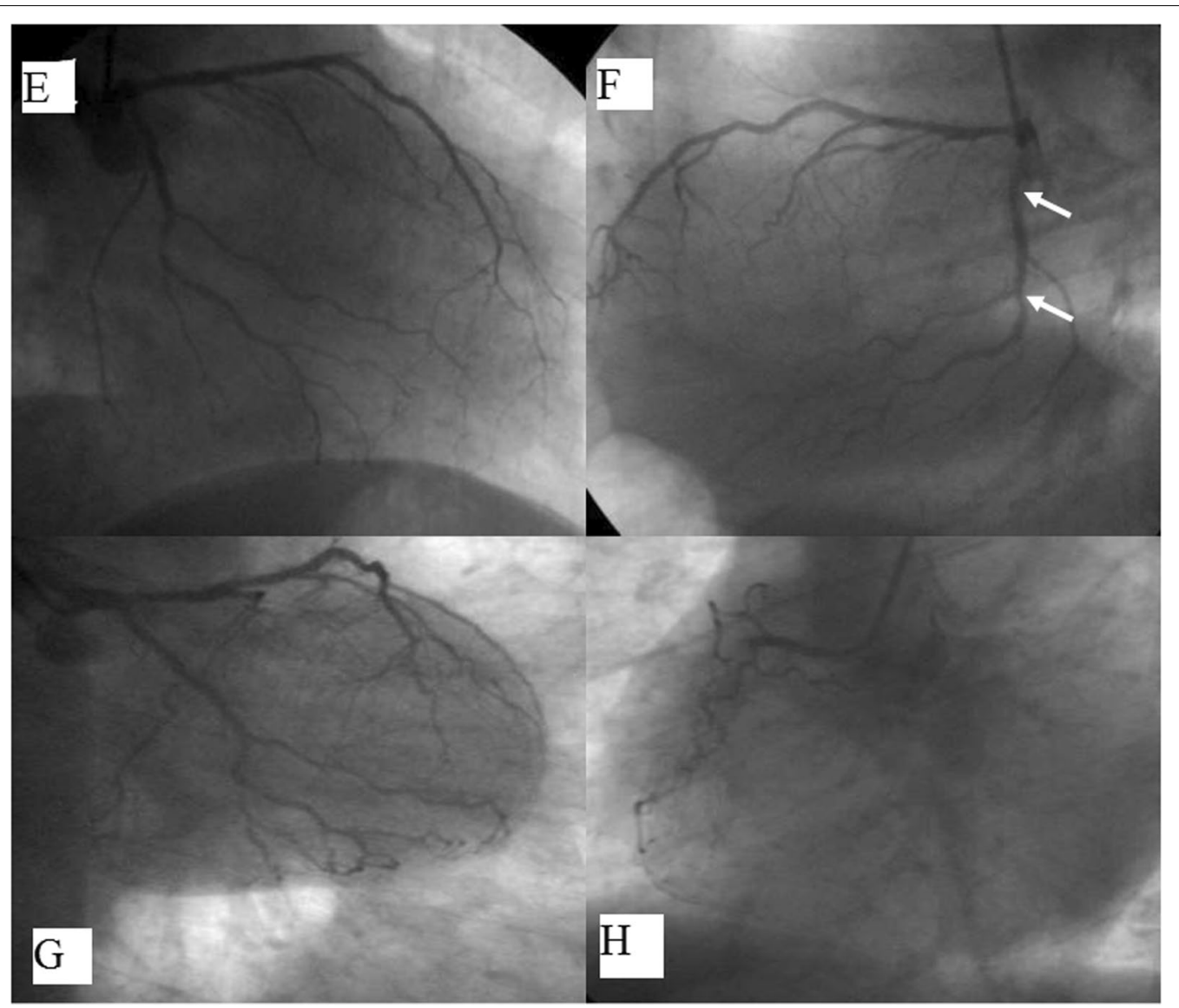

Fig. 2 continued

\section{Discussion}

Conventional coronary angiography is considered the gold standard in the assessment of coronary arteries. However, the continued advancement in CT technology, as well as the development of a new CAD-RAD system, have increased the dependence on CT coronary angiography as a gatekeeper for the coronary artery disease assessment with limitation of the coronary artery angiography to cases that require revascularization [9].

This study included forty-eight patients, 24 patients (50\%) had normal angiograms, 8 patients $(16.67 \%)$ had a non-significant disease and the other 16 patients (33.33\%) had a significant disease, which was similar to the previous study done by Raff et al. [10] that showed 35\% of the patients with normal angiograms, $28 \%$ with non-significant coronary disease and $37 \%$ had a significant disease. The right-sided dominance of coronary circulation was most commonly seen $(83.33 \%)$ compared to $(62.5 \%)$ at Madhok and Aggarwal et al. [11].

In the 48 patients, only two patients showed significant lesions on the conventional angiography images and were missed or underestimated on CT coronary angiography
(7.2\%). These lesions were at the small branch arteries. That can be explained by the fact that despite the improvement in the temporal and spatial resolution of CT reaching 0.4 to $0.5 \mathrm{~mm}$ it is still not comparable to conventional coronary angiography which is 0.1 to $0.2 \mathrm{~mm}$ [12]. On the other hand, only two patients showed lesions that were overestimated at the CT angiography as significant yet they proved to be insignificant at conventional angiography. The over-estimated lesions at $\mathrm{CT}$ angiography are usually attributed to the presence of extensive atheromatous calcification of the vessel wall with subsequent beam hardening artefact that gave the false impression of being significant. The false positive and false negative results in this study were lower than that of Raff et al. [10] study that showed about $17 \%$ false negative and $20 \%$ false positive, this difference is likely due to the continuous advancement in CT technology and post-processing algorithms aiming at improving the resolution and limiting the effect of beam hardening artefacts.

In the current study, the MDCT coronary angiography had an overall sensitivity of $92.9 \%$, which was higher than 


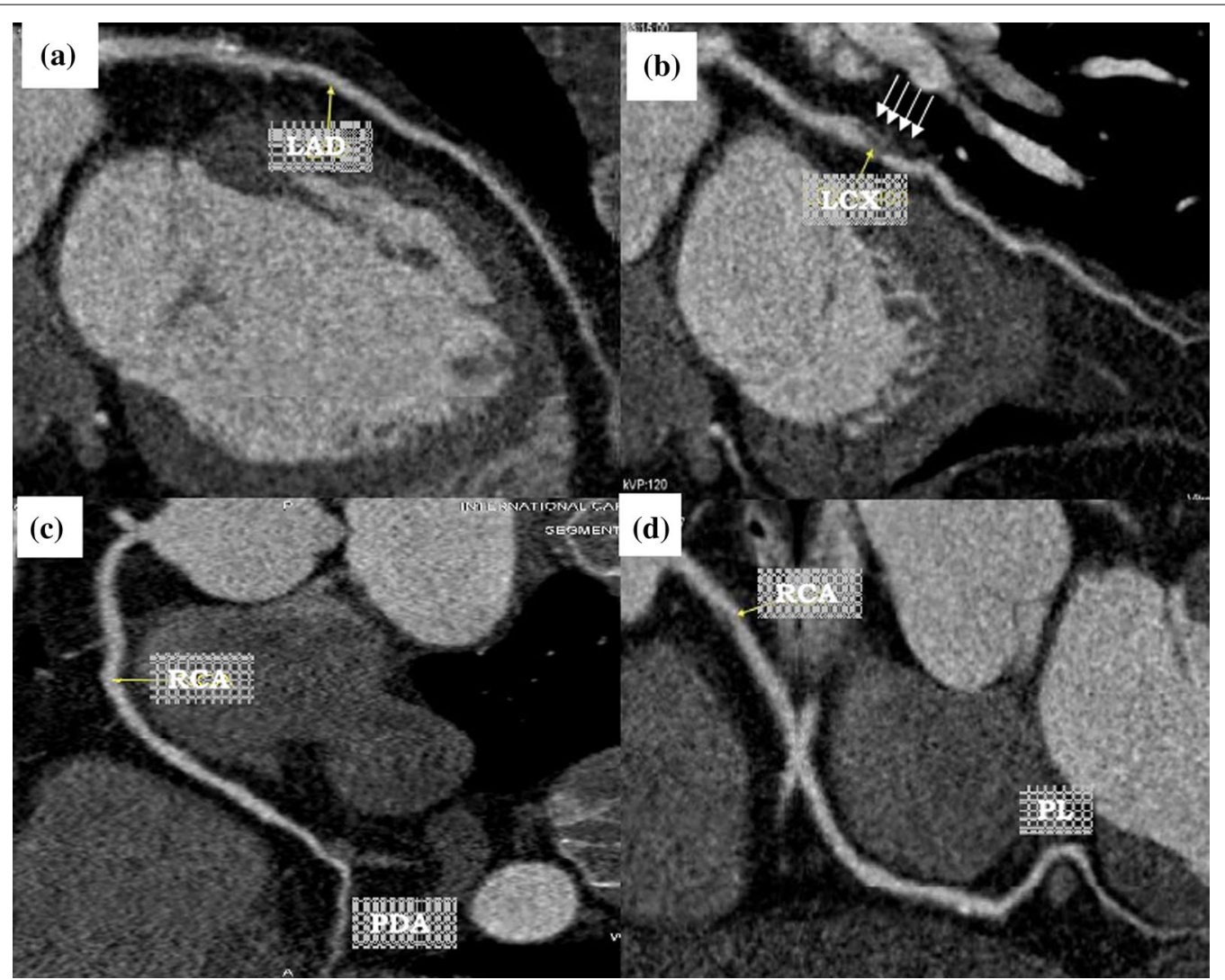

Fig. 3 A 48-year-old male patient with recurrent attacks of retro-sternal compressing pain. A-D CT angiographic showed mid-LCX segment significant stenotic segment (multiple arrows) with mild diffuse atherosclerotic changes of the rest of the coronary arteries with no significant lesions (CAD-RADS 4). E-H Conventional angiographic findings showed a significant stenotic lesion at mid-LCX segment (arrows) with mild diffuse atherosclerotic changes of the rest of the coronary arteries with no significant lesions in complete agreement with the CT findings

the value reported in the previous studies done by Raff et al. [10], Madhok and Aggarwal et al. [11] and Leber et al. [13] that reported sensitivity of $86 \%, 87 \%$ and $82 \%$, respectively. The specificity of the MDCT coronary angiography in this study was $90 \%$, near to the specificity (95\%) which was reported in Raff et al. [10], Madhok and Aggarwal et al. [11]. Leber et al. [13] studies. The positive predictive value in this study was $92.9 \%$ being relatively higher compared to the values reported by Raff et al. [10], Madhok and Aggarwal et al. [11] and Leber et al. [13] which were $66 \%, 75 \%$ and $72 \%$, respectively. Our negative predictive value was $90 \%$ which was similar to the values of Raff et al. [10], Madhok and Aggarwal et al. [11] and Leber et al. [13] who reported negative predictive value of $98 \%, 98 \%$ and $97 \%$, respectively. The higher value of sensitivity and the positive predictive value in this study is partially explained by the ability of the new CT generation which correctly identify the smaller lesions in the distal segments.

On the contrary, the sensitivity, specificity, negative predictive values and positive predictive values in this study were relatively lower than values noted in Basha et al. [14] study that were $100 \%, 98 \%, 98 \%$ and $100 \%$, respectively; this can be attributed to a large number of significant lesions in Basha et al. [14] study that represented about $45 \%$ of the sample size which could represent a form of selection bias.

Regarding the per-segment evaluation of the coronary arteries, the agreement between CT angiography and the conventional angiography was an excellent agreement for the left main trunk at its all segments, similar to that of Waseem et al. [15] and Mannan et al. [16] who revealed excellent agreement $(k=1)$.

As regards the left anterior descending artery at its proximal, mid- and distal segments as well as the second diagonal branch there was excellent agreement between conventional and CT angiography in the current study with good agreement seen at the first diagonal branch $(K=0.765)$, similar results were also noted in two different studies done by Waseem et al. [15] and Mannan et al. [16] with $K$ range from 0.788 to 1 . 


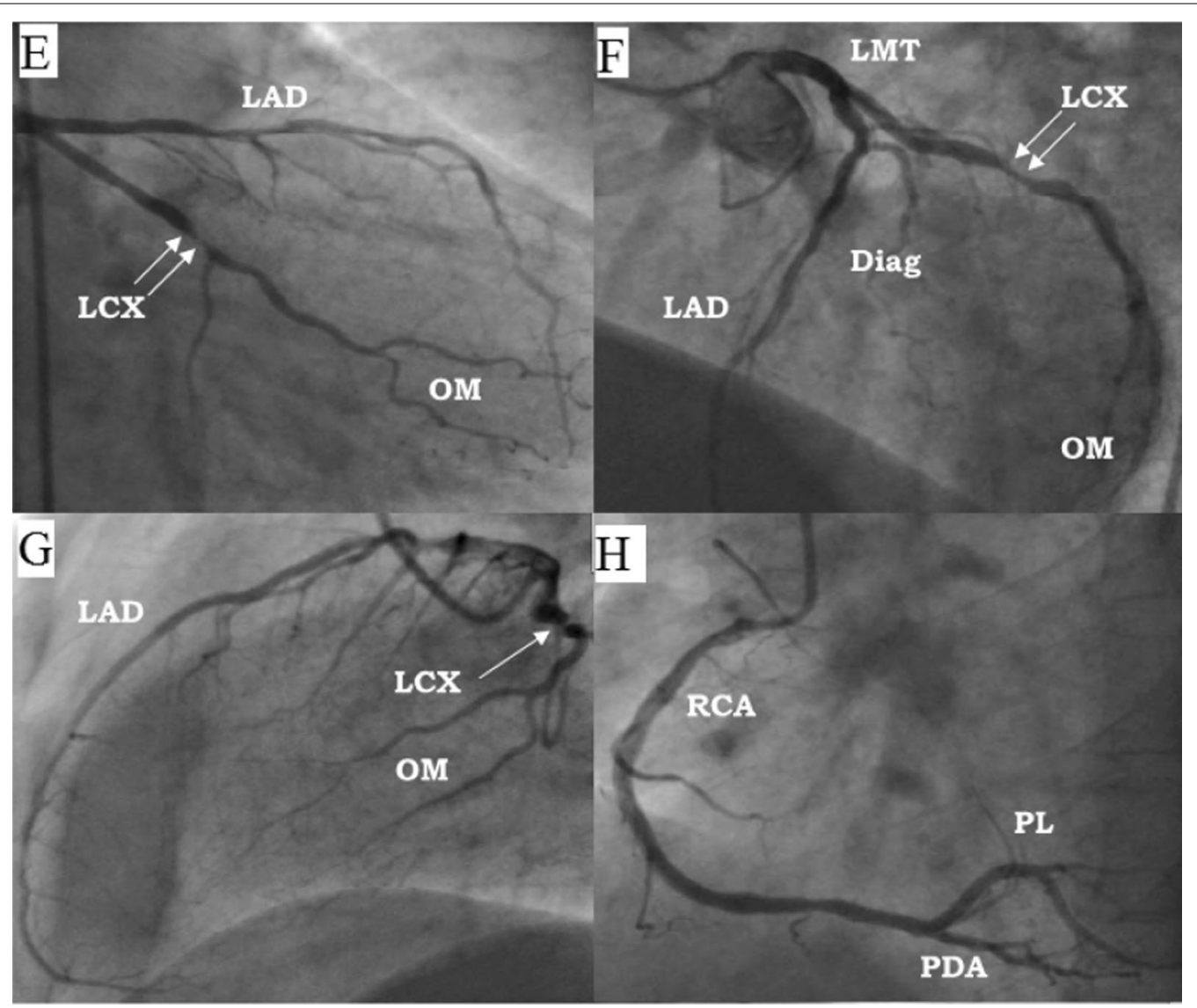

Fig. 3 continued
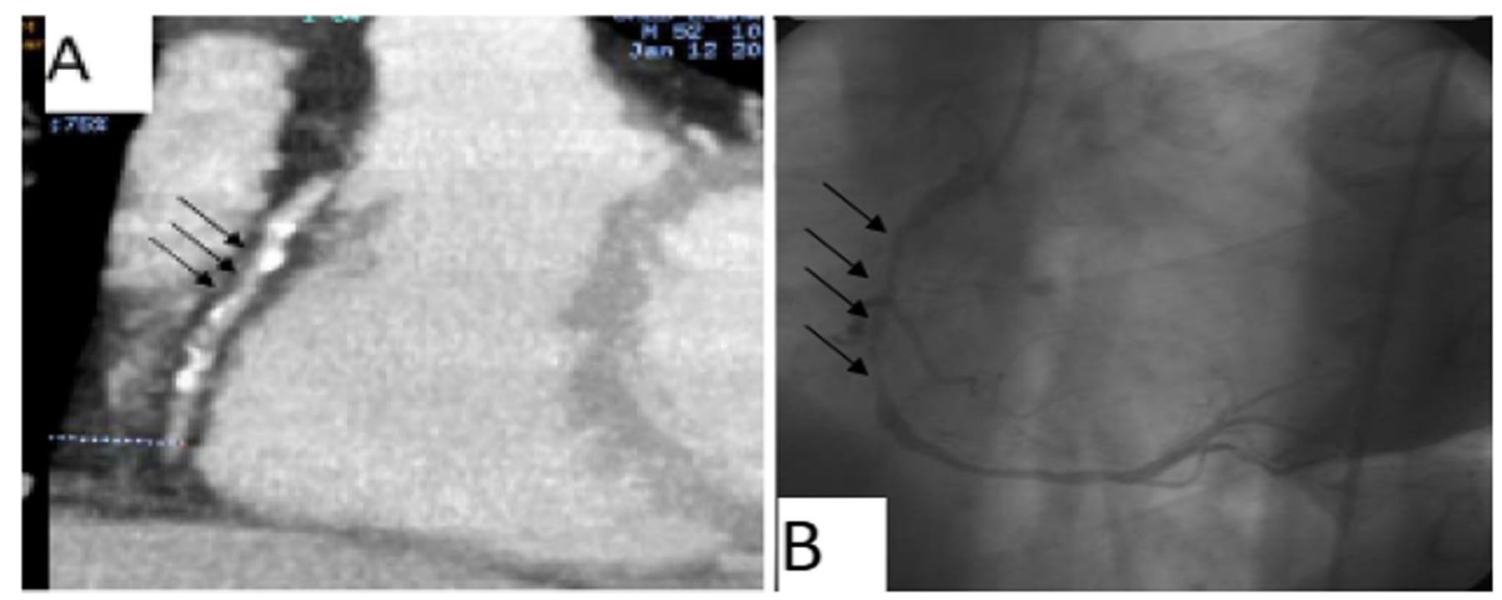

Fig. 4 A 61-year-old male patient complaining of recurrent attacks of stabbing chest pain radiating to the left arm. A CT angiography showed RCA long partially calcified lesions (three black arrows) (CAD-RADS 4). B Conventional angiography images showed RCA mid-segment long stenotic lesions (multiple arrows) in complete agreement with the CT findings 
Table 3 Analysis of the plaque types in the conventional and CT angiography

\begin{tabular}{lclll}
\hline Plaque type & CT angiography & $\begin{array}{l}\text { Conventional } \\
\text { angiography }\end{array}$ & kappa & $\boldsymbol{p}$ value \\
\hline Calcified plaques & $17(35.4 \%)$ & $13(27.1 \%)$ & 0.601 & $0.004^{*}$ \\
No plaques & $25(52.1 \%)$ & $35(72.9 \%)$ & & \\
$\begin{array}{l}\text { Non-calcific } \\
\text { plaques }\end{array}$ & $6(12.5 \%)$ & & \\
\hline K: kappa coefficient & & & \\
*Statistically significant $(\boldsymbol{p}<0.05)$ & &
\end{tabular}

Table 4 Comparison between the Computed Tomography (CT) coronary angiography by using (CAD-RADS) and the conventional coronary angiography in all patients (per-patient analysis)

\begin{tabular}{|c|c|c|c|c|}
\hline \multirow{3}{*}{$\begin{array}{l}\text { Computed CT } \\
\text { angiography }\end{array}$} & \multicolumn{4}{|c|}{ Conventional angiography } \\
\hline & \multicolumn{2}{|c|}{ Non-significant $(n=20)$} & \multicolumn{2}{|c|}{ Significant $(n=28)$} \\
\hline & No & $\%$ & No & $\%$ \\
\hline Non-significant & 18 & 90 & 2 & 7.2 \\
\hline Significant & 2 & 10 & 26 & 92.8 \\
\hline Sensitivity & $92.9 \%$ & & & \\
\hline Specificity & $90 \%$ & & & \\
\hline Accuracy & $91.7 \%$ & & & \\
\hline PPV & $92.9 \%$ & & & \\
\hline NPV & $90 \%$ & & & \\
\hline
\end{tabular}

An excellent agreement was noted for the left circumflex artery (LCX) at its proximal, mid-, distal segments and the obtuse marginal branch, Waseem et al. [15] and
Mannan et al. [16] also found an excellent agreement for the LCX and OM branch yet with $k$ range from 0.846 to 0.9 .

In this study, there was excellent agreement $(K=1)$ in the proximal and mid-segments of the right coronary artery, as well as the posterior descending artery, with a good agreement in the distal segment of the RCA $(K=0.782)$, which was similar to the results of Waseem et al. [15] and Mannan et al. [16] who reported excellent agreement for all RCA segment and PDA but the distal segment showed a good agreement with $k=0.765$.

The CAD-RADS classification has several limitations, the most important is that it does not account for the total plaque volume which is found to be a strong predictor for acute coronary syndrome and important discriminator from stable coronary artery disease $[17,18]$, another important limitation is that it is not applicable for vessels less than $1.5 \mathrm{~mm}$ which limits its ability is the assessment of diagonal branches distal segments [19]. Another important drawback is that due to potentially complex conditions in coronary anatomy, CADRADS might not appropriately classify patients with coronary anomalies. These patients continue to require comprehensive reports and individual management recommendations [20]. Apart from these limitations, the standardized reporting algorithm ensures detailed and reliable reporting, as well as clear communication between clinicians, radiologists and patients [21-24].

The limitation of our study was that we included a relatively small number of patients. Also as we were focusing upon comparing the results of CT with conventional angiography, we did not include incidental

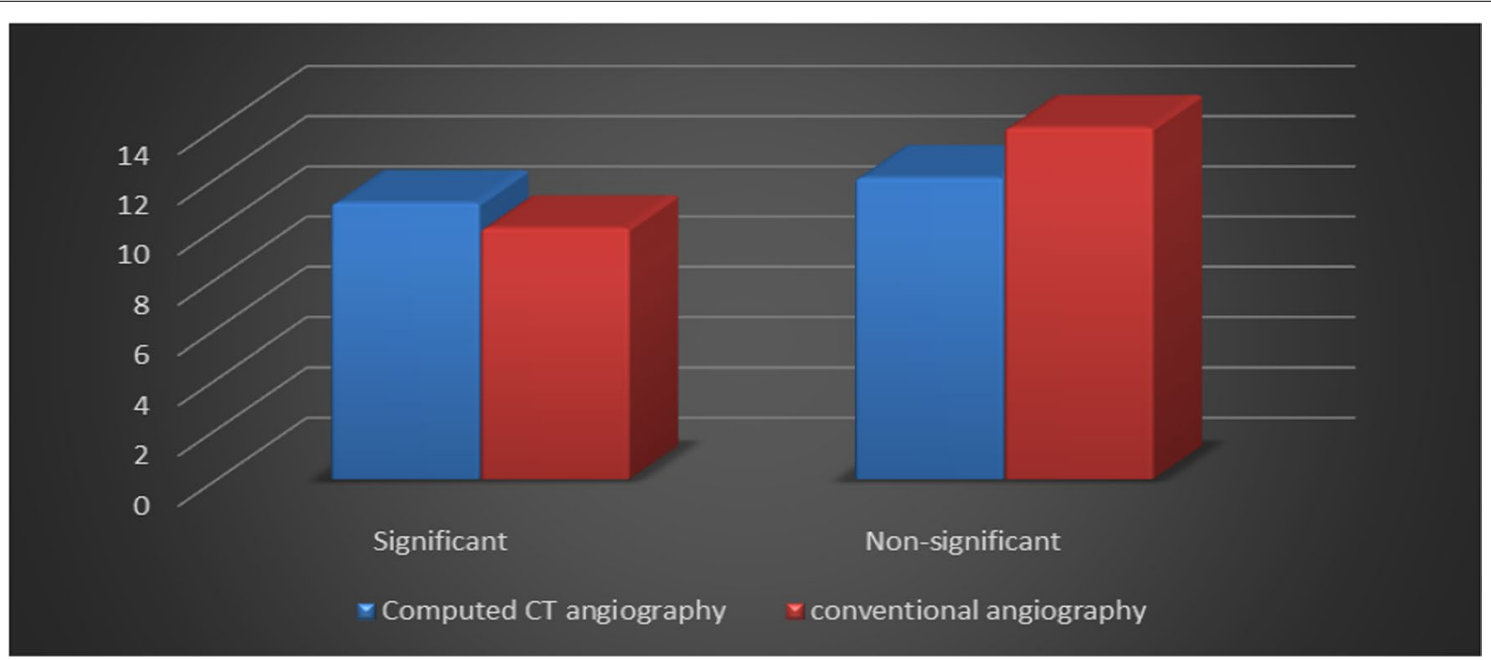

Fig. 5 Comparison between the computed tomography (CT) coronary angiography by using "coronary artery disease-reporting and data system (CAD-RADS)" and the conventional coronary angiography in all patients (per-patient analysis) 
coronary anomalies, calcium scoring and incidental non-coronary and extra cardiac findings in our results.

\section{Conclusions}

The CAD-RADS algorithm and conventional angiography had a perfect inter-method agreement, thus supporting the concept that the coronary $\mathrm{CT}$ angiography using the CAD-RADS could be considered the primary imaging modality for coronary artery disease, with invasive conventional coronary angiography reserved for cases requiring intervention.

\begin{abstract}
Abbreviations
CAD: Coronary artery disease; CCTA: Coronary computed tomography angiography; CAD-RADS: Coronary artery disease reporting and data system; CT: Computed tomography; ICA: Invasive conventional angiography; LMT: Left main trunk; $k$ : Kappa coefficient; LAD: Left anterior descending artery; D1: First diagonal; D2: Second diagonal; LCx: Left circumflex artery; RCA: Right coronary artery; OM: Obtuse marginal; PDA: Posterior descending artery; PPV: Positive predictive value; NPV: Negative predictive value.
\end{abstract}

\section{Acknowledgements}

Not applicable.

\section{Authors' contributions}

ME: Results and statistics, manuscript writing. MS: Idea of the research, revising the manuscript. AM: Idea of the research, collecting cases. AS: Results and statistics, manuscript writing, revising the manuscript. All authors read and approved the final manuscript.

\section{Funding}

No Funds, sponsorship or financial support to be disclosed.

\section{Availability of data and materials}

The datasets used and/or analyzed during the current study are available from the corresponding author on reasonable request.

\section{Declarations}

Ethics approval and consent to participate

This study was approved by the Research Ethics Committee of the Faculty of Medicine at Ain shams University in Egypt (FWA 000017585); Reference Number of approval: R67/2020. Written informed consent was signed by all patients who participated in this study.

\section{Consent for publication}

Not applicable.

\section{Competing interests}

The authors declare that they have no competing interests.

Received: 18 November 2021 Accepted: 16 January 2022 Published online: 01 February 2022

\section{References}

1. Mack M, Gopal A (2016) Epidemiology, traditional and novel risk factors in coronary artery disease. Heart Fail Clin 12:1-10

2. Sabri N (1994) Complications of cardiac catheterization, coronary angiography, and coronary interventions. J Invasive Cardiol 6(9):300-305

3. Roifman I, Rezai MR, Wijeysundera HC et al (2015) Utilization of cardiac computed tomography angiography and outpatient invasive coronary angiography in Ontario, Canada. J Cardiovasc Comput Tomogr 9:567-571
4. Cury RC, Abbara S, Achenbach S et al (2016) CAD-RADS(TM) coronary artery disease-reporting and data system. An expert consensus document of the society of cardio-vascular computed tomography (SCCT), the American College of radiology (ACR) and the North American society for cardiovascular imaging (NASCI). Endorsed by the American College of cardiology. J Cardiovasc Comput Tomogr 10:269-281

5. Douglas PS, Hoffmann U, Patel MR et al (2015) Outcomes of anatomical versus functional testing for coronary artery disease. N Engl J Med 372:1291-1300

6. SCOT-HEART investigators (2015) CT coronary angiography in patients with suspected angina due to coronary heart disease (SCOT-HEART): an open label, parallel-group, multicentre trial. Lancet 385:2383-2391

7. Goldstein JA, Chinnaiyan KM, Abidov A et al (2011) The CT-STAT (coronary computed tomographic angiography for systematic triage of acute chest pain patients to treatment) trial. J Am Coll Cardiol 58:1414-1422

8. Chandrashekhar Y, Min JK, Hecht H et al (2016) CAD-RADS: a giant first step toward a common lexicon. JACC Cardiovasc Imaging 9:1125-1129

9. Moscariello A, Vliegenthart R, Schoepf UJ et al (2012) Coronary CT angiography versus conventional cardiac angiography for therapeutic decision making in patients with high likelihood of coronary artery disease. Radiology 265(2):385-392

10. Raff GL, Gallagher MJ, O’Neill WW et al (2005) Diagnostic accuracy of noninvasive coronary angiography using 64-slice spiral computed tomography. J Am Coll Cardiol 46(3):552-557

11 Madhok R, Aggarwal A (2014) Comparison of 128-slice dual-source CT coronary angiography with invasive coronary angiography. J Clin Diagn Res 8(6):RC08

12. Sabarudin A, Sun Z (2013) Coronary CT angiography: diagnostic value and clinical challenges. World J Cardiol 5(12):473-483. https://doi.org/10.4330/ wjc.v5.i12.473

13. Leber AW, Johnson T, Becker A et al (2007) Diagnostic accuracy of dual-source multi-slice CT-coronary angiography in patients with an intermediate pretest likelihood for coronary artery disease. Eur Heart J 28(19):2354-2360

14. Basha MA, Aly SA, Ismail AA et al (2019) The validity and applicability of CADRADS in the management of patients with coronary artery disease. Insights Imaging 10(1):117

15. Waseem A, Hafeez DM, Dar Ml et al (2016) Diagnostic utility of gadolinium enhanced coronary CT angiography compared to conventional invasive coronary angiography in detection of obstructive coronary artery disease. J Clin Exp Cardiol 7(479):2

16. Mannan N, Basher MA, Jahan MU et al (2014) Comparison of coronary CT angiography with conventional coronary angiography in the diagnosis of coronary artery disease. Bangladesh Med Res Counc Bull 40(1):31-35

17. Pflederer T, Marwan M, Schepis T et al (2010) Characterization of culprit lesions in acute coronary syndromes using coronary dual-source CT angiography. Atherosclerosis 211(2):437-444

18. Dey D, Achenbach S, Schuhbaeck A et al (2014) Comparison of quantitative atherosclerotic plaque burden from coronary $\mathrm{CT}$ angiography in patients with first acute coronary syndrome and stable coronary artery disease. J Cardiovasc Comput Tomogr 8(5):368-374

19. Canan A, Ranganath $P$, Goerne $\mathrm{H}$ et al (2020) CAD-RADS: pushing the limits. Radiographics 40(3):629-652. https://doi.org/10.1148/rg.2020190164

20. Foldyna B, Szilveszter B, Scholtz JE, Banerji D et al (2018) CAD-RADS—a new clinical decision support tool for coronary computed tomography angiography. Eur Radiol 28(4):1365-1372. https://doi.org/10.1007/ s00330-017-5105-4

21. Abdelrahman AS, Ashour MMM, Abdelaziz TT (2020) Predictive value of neck imaging reporting and data system (NIRADS) in CECT/CEMRI of laryngeal and oral cavity squamous cell carcinoma. Egypt J Radiol Nucl Med $51: 241$

22. Abdelaziz TT, Abdel Razk AAK, Ashour MMM et al (2020) Interreader reproducibility of the neck imaging reporting and data system (NI-RADS) lexicon for the detection of residual/recurrent disease in treated head and neck squamous cell carcinoma (HNSCC). Cancer Imaging 20:61

23. Abdelrahman AS, Abdel-Rahman AS, Taha NM (2021) The role of functional imaging; DWI, ADC and 18F-FDG PET/CT in the evaluation of HCC treatment response after transarterial chemoembolization. Egypt J Radiol Nucl Med 52:217 
24. Elrefaey Hasan BM, Abd ElHamid HAE, Khater NH et al (2021) Role of DWl in evaluation of HCC after radiofrequency ablation compared to dynamic MRI using MRI (3 T). Egypt J Radiol Nucl Med 52:267

\section{Publisher's Note}

Springer Nature remains neutral with regard to jurisdictional claims in published maps and institutional affiliations.

\section{Submit your manuscript to a SpringerOpen ${ }^{\circ}$ journal and benefit from:}

- Convenient online submission

- Rigorous peer review

- Open access: articles freely available online

- High visibility within the field

- Retaining the copyright to your article

Submit your next manuscript at $\boldsymbol{\nabla}$ springeropen.com 